\title{
A Systematic Review of Intravenous $\beta$-Hydroxybutyrate Use in Humans - A Promising Future Therapy?
}

\begin{abstract}
Hayden White ${ }^{1,2 *}$, Aaron J. Heffernan ${ }^{1,2}$, Simon Worrall ${ }^{3}$, Alexander Grunsfeld ${ }^{4}$ and Matt Thomas ${ }^{5}$

${ }^{1}$ Department of Intensive Care Medicine, Logan Hospital, Meadowbrook, QLD, Australia, ${ }^{2}$ School of Medicine, Griffith University, Southport, QLD, Australia, ${ }^{3}$ Department of Biochemistry and Molecular Biology, Faculty of Science, School of Chemistry and Molecular Biosciences, The University of Queensland, Brisbane, QLD, Australia, ${ }^{4}$ Department of Neurosciences, Eastern Virginia Medical School, Sentara Healthcare, Norfolk, VA, United States, ${ }^{5}$ Department of Intensive Care Medicine, North Bristol NHS Trust, School of Physiology, Pharmacology and Neuroscience, University of Bristol, Bristol, United Kingdom
\end{abstract}

Therapeutic ketosis is traditionally induced with dietary modification. However, owing to the time delay involved, this is not a practical approach for treatment of acute conditions such as traumatic brain injury. Intravenous administration of ketones would obviate this problem by rapidly inducing ketosis. This has been confirmed in a number of small animal and human studies. Currently no such commercially available product exists.

OPEN ACCESS

Edited by:

Victoria Bunik,

Lomonosov Moscow State

University, Russia

Reviewed by:

Marko Cincović,

University of Novi Sad, Serbia

Yu Qian,

University of Texas MD Anderson

Cancer Center, United States

*Correspondence: Hayden White

hayden.white@health.qld.gov.au

Specialty section:

This article was submitted to Translational Medicine,

a section of the journal

Frontiers in Medicine

Received: 13 July 2021

Accepted: 24 August 2021

Published: 21 September 2021

Citation:

White $H$, Heffernan AJ, Worrall S, Grunsfeld A and Thomas M (2021) A

Systematic Review of Intravenous $\beta$-Hydroxybutyrate Use in Humans -

A Promising Future Therapy?

Front. Med. 8:740374.

doi: 10.3389/fmed.2021.740374
The aim of this systematic review is to review the safety and efficacy of intravenous beta-hydroxybutyrate. The Web of Science, PubMed and EMBASE databases were searched, and a systematic review undertaken. Thirty-five studies were included. The total beta-hydroxybutyrate dose ranged from 30 to $101 \mathrm{~g}$ administered over multiple doses as a short infusion, with most studies using the racemic form. Such dosing achieves a beta-hydroxybutyrate concentration $>1 \mathrm{mmol} / \mathrm{L}$ within $15 \mathrm{~min}$. Infusions were well tolerated with few adverse events. Blood glucose concentrations occasionally were reduced but remained within the normal reference range for all study participants. Few studies have examined the effect of intravenous beta-hydroxybutyrate in disease states. In patients with heart failure, intravenous beta-hydroxybutyrate increased cardiac output by up to $40 \%$. No studies were conducted in patients with neurological disease. Intravenous beta-hydroxybutyrate has been shown to increase cerebral blood flow and reduce cerebral glucose oxidation. Moreover, beta-hydroxybutyrate reduces protein catabolism and attenuates the production of counter-regulatory hormones during induced hypoglycemia. An intravenous beta-hydroxybutyrate formulation is well tolerated and may provide an alternative treatment option worthy of further research in disease states.

Keywords: ketones, acetoacetate, beta-hydroxybutyrate (BHB), ketosis, intravenous

\section{INTRODUCTION}

3-beta-Hydroxybutyric acid (beta-hydroxybutyrate; BHB), acetoacetic acid (acetoacetate; AcAc), and acetone (Supplementary Figure 1) are collectively referred to as "ketone bodies." In humans, AcAc and BHB accumulate when carbohydrate availability is low, due to the oxidation of fatty acids released from stores in adipose tissue to serve as an alternative energy source for cellular respiration 
(Supplementary Figure 2). The predominant ketone bodies, $\mathrm{AcAc}$, and BHB are energy-rich and can be used to export usable energy to extra-hepatic tissues. This means that when blood glucose concentrations are low, the use of ketone bodies spares glucose $(1,2)$ for use by tissues such as erythrocytes and the brain that are obligate glucose users. Under these conditions the large amounts of fatty acids being released from the adipose stores cannot, unlike most other tissues, be directly used by the brain to generate energy. However, the ketone bodies generated from the excessive production of acetyl-CoA in the liver, can be used as an alternative source of energy, and can provide about $60-70 \%$ of the brain's energy requirements during extended starvation.

With this in mind, ketosis is usually associated with a pathological state, such as diabetic ketoacidosis, starvation or alcohol intoxication. Moreover, the ratio of acetoacetate to BHB (arterial ketone body ratio) reflects the efficiency of cellular metabolism (mitochondrial redox state) (3-5), and therefore can be viewed as a concerning marker of cellular hypoxia. Despite this, the intentional induction of ketosis with prolonged dietary modifications, such as ketogenic diets, may have a therapeutic role. For example, the use of such diets in two recent randomizedcontrolled trials showed improved seizure control in epileptic children. Ketogenic diets have also been examined in other pathological conditions including heart disease, neurological disorders such as Alzheimer disease, Parkinson disease, motor neuron disease, stroke, traumatic brain injury and migraine (6$11)$.

Importantly, the role of therapeutic ketosis in acute pathological states such as traumatic brain injury, stroke, heart failure and other diseases has been less extensively investigated due to the lack of a commercially available supply of intravenous BHB. Rapid induction of ketosis with intravenous BHB administration may have a therapeutic role in optimizing cellular respiration in disease states. The purpose of this systematic review is to examine the potential safety, pharmacokinetics and physiological outcomes of patients receiving intravenous BHB formulations. Whereas several reviews on ketone bodies and their utility as therapeutic agents have been published the authors believe this to be the first systematic review to focus solely on the use of BHB as an intravenous therapeutic agent.

\section{METHODS}

\section{Search Strategy}

Studies were retrieved from the PubMed, Web of Science, and EMBASE databases. The following search phrase was used: [(keton*) OR (hydroxybutyrate*) OR (beta-hydroxybutyr*) OR (3-hydroxybutyr*) OR (sodium-hydroxybutyr*)] AND

Abbreviations: AcAc, acetoacetate; acetyl CoA, coenzyme A; ADR, adverse drug reaction; $\mathrm{BHB}, \beta$-hydroxybutyrate; $\mathrm{BMI}$, body mass index; $\mathrm{CI}$, continuous infusion; CO, cardiac output; DKA, diabetic ketoacidosis; EG, euglucemic; FFA, free fatty acids; GFR, glomerular filtration rate; CNS, central nervous system; GH, growth hormone; HFrEF, heart failure with reduced ejection fraction; HR, heart rate; NYHA, New York Heart Association; IDDM, insulin dependent diabetes mellitus; NEFA, non-esterified fatty acids; T1DM, type 1 or insulin-dependent Diabetes mellitus;T2DM, type 2 or non-insulin-dependent Diabetes mellitus; SVR, systemic vascular resistance; VT, ventricular tachycardia. [(intraven*) OR (infusi*) OR (intra-veno*)] and limited using the relevant study filter to include only human participants where possible. Published studies from 1946 until the 27th of October 2020 were included. A bibliography search of included articles identified for full text review was also performed. Other articles were identified from a search of the author's extensive records.

\section{Inclusion and Exclusion Criteria}

Published peer-reviewed manuscripts were included if they included human subjects who received any intravenous formulation of BHB. Articles including animals, subjects receiving other ketone formulations, or administration of BHB by a route other than intravenous were excluded. Studies with insufficient description of BHB administration were also excluded. Articles written in a language other than English were excluded. Articles for full reference review and data extraction were selected based on the abstract of articles identified in the search. A summary of the review is presented in the Preferred Reporting Items for Systematic Reviews and Meta-Analyses (PRISMA) flow chart (Supplementary Figure 3).

\section{Data Extraction}

The following study details were extracted, where available, from the included studies: study participant demographic details (population type, number of participants, weight and body mass index $[\mathrm{BMI}]$ ), BHB formulation, fasting status, use of insulin, and total dose of BHB. Outcome variables extracted included $\mathrm{BHB}$ concentration, any relevant study outcome variable, and any adverse events experienced during the study.

Article identification, evaluation, and data extraction were performed by two independent reviewers ( $\mathrm{AH}$ and $\mathrm{HW}$ ). When disagreements occurred, resolution was mediated by consultation with other authors.

\section{RESULTS}

\section{BHB in Cardiac Disease}

Animal studies associating ketone administration with improved cardiac performance has stimulated interested in human studies. Results for studies involving cardiovascular endpoints are summarized in Table 1. Two studies were identified. Gormsen et al., demonstrated a 75\% increased myocardial blood flow and decreased glucose uptake in healthy participants following intravenous ketone administration (13). More recently, Nielsen et al. investigated the effects of BHB infusions on patients with chronic heart failure (New York Heart Association Classification II-III) with reduced ejection fraction (14). Cardiac output increased by $40 \%(2.0 \pm 0.3 \mathrm{~L} / \mathrm{min})(p<0.001)$, left ventricular ejection fraction $8 \%$, stroke volume, heart rate and mixed venous oxygen saturation all demonstrated significant increases while systemic venous resistance decreased by $30 \%$. Mean arterial pressure remained stable throughout. Moreover, similar to the Gormsen et al. study, myocardial blood flow also increased in this patient population. A follow up study where BHB concentrations were slowly increased to $\sim 50 \%$ of the previous study also confirmed an increase in cardiac output $(1.2 \pm 0.1 \mathrm{~L} / \mathrm{min} ; P$ $<0.001$ ) although less than the initial result. They concluded 
TABLE 1 | Ketone studies investigating cardiac outcomes.

\begin{tabular}{|c|c|c|c|c|c|c|c|c|c|c|c|c|}
\hline Year & Author & Study & Population & $\begin{array}{l}\text { No. } \\
\text { participants }\end{array}$ & Formulation & Fasting & Intervention & $\begin{array}{l}\text { Total } \\
\text { average } \\
\text { BHB dose }\end{array}$ & $\begin{array}{l}\text { Insulin } \\
\text { used }\end{array}$ & Findings overview & $\begin{array}{l}\text { Concentration } \\
\text { BHB }\end{array}$ & ADR \\
\hline 1993 & $\begin{array}{l}\text { Vanoverschelde } \\
\text { et al. (12) }\end{array}$ & Cross-over & Healthy & 6 & $\begin{array}{l}\text { Dextro-3- } \\
\text { OHB } \\
576 \mathrm{mM} \text {. }\end{array}$ & Fasting & $\begin{array}{l}40 \\
\mu \mathrm{mol} / \mathrm{kg} / \mathrm{min} \\
\text { for } 20 \mathrm{~min} \\
\text { followed by } \\
17 \\
\mu \mathrm{mol} / \mathrm{kg} / \mathrm{min}\end{array}$ & & No & $\begin{array}{l}\text { Decreased }{ }^{11} \mathrm{C} \text {-Palmitate } \\
\text { myocardial uptake indicating } \\
\text { reduced fatty acid oxidation. }\end{array}$ & $\begin{array}{l}0.06 \pm 0.05 \text { to } \\
1.15 \pm 0.30 \mathrm{mM}\end{array}$ & Nil noted \\
\hline 2017 & $\begin{array}{l}\text { Gormsen et al. } \\
\text { (13) }\end{array}$ & $\begin{array}{l}\text { Randomized } \\
\text { placebo- } \\
\text { controlled } \\
\text { single blinded } \\
\text { crossover }\end{array}$ & Healthy & 8 & $\begin{array}{l}\text { Racaemic } \\
\text { Na-3-OHB } \\
7.5 \%\end{array}$ & Fasting & $\begin{array}{l}0.18 \mathrm{~g} / \mathrm{kg} / \mathrm{h} \\
\text { for } 6.5 \mathrm{~h}\end{array}$ & & Yes - EG & $\begin{array}{l}\text { Increased myocardial blood } \\
\text { flow in all coronary artery } \\
\text { vascular territories ( } 75 \%) \\
\text { with an increased HR } \\
\text { potentially associated with } \\
\text { alkalosis and reduced } \\
\text { potassium. Myocardial } \\
\text { glucose uptake halved. }\end{array}$ & $\begin{array}{l}<50 \text { microM to } \\
3.78 \pm 0.47 \mathrm{mM} \\
\text { post-infusion }\end{array}$ & Nil noted \\
\hline 2019 & $\begin{array}{l}\text { Nielsen et al. } \\
\text { (14) }\end{array}$ & $\begin{array}{l}\text { Randomized } \\
\text { placebo- } \\
\text { controlled } \\
\text { single blinded } \\
\text { crossover }\end{array}$ & $\begin{array}{l}\text { HFrEF NYHA } \\
\text { Class II-III + } \\
\text { Healthy }\end{array}$ & $\begin{array}{l}\text { Study 1: 24/10 } \\
\text { HFrEF/controls; } \\
\text { Study 2: } 8 \\
\text { HFrEF }\end{array}$ & $\begin{array}{l}\text { Racaemic } \\
\mathrm{Na}-3-\mathrm{OHB} \\
7.5 \%+\mathrm{KCl} \\
60 \mathrm{mM}\end{array}$ & Fasting & $\begin{array}{l}\text { Study 1: } 0.18 \\
\mathrm{~g} / \mathrm{kg} / \mathrm{h} \text { for } 3 \mathrm{~h} ; \\
\text { Study 2: } \\
0.045 \mathrm{~g} / \mathrm{kg} / \mathrm{h} \\
\text { for } 2 \mathrm{~h} \text { then } \\
\text { separately } \\
0.09 \mathrm{~g} / \mathrm{kg} / \mathrm{h} \\
\text { for } 2 \mathrm{~h} \text { S Study } \\
3: 0.18 \\
\mathrm{~g} / \mathrm{kg} / \mathrm{h} \text { for } 3 \mathrm{~h}\end{array}$ & & Yes - EG & $\begin{array}{l}\text { Dose dependent increase in } \\
\mathrm{CO} \text { in patients with HF, } \\
\text { potentially related to } \\
\text { decreased SVR. }\end{array}$ & $\begin{array}{l}\text { Study } 1: 0.4 \pm \\
0.3 \mathrm{mM} \text { baseline to } \\
3.3 \pm 0.4 \mathrm{mM} \text { after } \\
3 \mathrm{~h} \text { infusion. Study } \\
2:<0.1 \mathrm{mM} \text { to } 0.7 \\
\pm 0.1 \mathrm{mM} \\
\text { following } 0.045 \\
\mathrm{~g} / \mathrm{kg} / \mathrm{h} ; 1.6 \pm \\
0.3 \mathrm{mM} \text { following } \\
0.09 \mathrm{~g} / \mathrm{kg} / \mathrm{h} \text {. Study } \\
3: 0.4 \pm 0.3 \mathrm{mM} \text { to } \\
3.4 \pm 0.6 \mathrm{mM} \text {. }\end{array}$ & $\begin{array}{l}\text { Asymptomatic VT } \\
\text { in one patient with } \\
\text { a history of VT. } \\
\text { Two episodes of } \\
\text { nausea (one } \\
\text { during placebo, } \\
\text { other following } \\
\text { infusion of } \\
\text { 3-OHB). }\end{array}$ \\
\hline
\end{tabular}

$A D R$, adverse drug reaction: $B H B$, beta-hydroxybutyrate; $C l$, continuous infusion: $C O$, cardiac output: $E G$, euglycaemic clamp; FFA, free fatty acids; NEFA, non-esterified fatty acids; GFR glomerular filtration rate; GH, growth hormone; HFrEF, heart failure with reduced ejection fraction; HR, heart rate; NYHA New York Heart Association; SVR, systemic vascular resistance; T1DM, type 1 or insulin-dependent Diabetes mellitus; T2DM, type 2 or non-insulin-dependent Diabetes mellitus; $V T$, ventricular tachycardia.

Data presented as mean \pm standard error 
TABLE 2 | Ketone studies investigating central nervous system outcomes.

\begin{tabular}{|c|c|c|c|c|c|c|c|c|c|c|c|c|}
\hline Year & Author (Ref) & Study & Population & $\begin{array}{l}\text { No. } \\
\text { participants }\end{array}$ & Formulation & Fasting & Intervention & $\begin{array}{l}\text { Total } \\
\text { average } \\
\text { BHB dose }\end{array}$ & Insulin used & Findings overview & Concentration BHB & ADR \\
\hline 1996 & $\begin{array}{l}\text { Hasselbalch } \\
\text { et al. (15) }\end{array}$ & $\begin{array}{l}\text { Randomized } \\
\text { crossover }\end{array}$ & Healthy & 8 & $\begin{array}{l}\text { Racaemic } \\
\text { BHB } 55 \\
\mathrm{mg} / \mathrm{mL} ; \mathrm{pH} \\
7.1\end{array}$ & $\begin{array}{l}4-5 \mathrm{~h} \\
\text { post } \\
\text { morning } \\
\text { meal }\end{array}$ & $\begin{array}{l}4-5 \\
\mathrm{mg} / \mathrm{kg} / \mathrm{min} \text { for } \\
3-3.5 \mathrm{~h}\end{array}$ & & No & $\begin{array}{l}\text { Global increase in cerebral } \\
\text { blood flow by 39\%. Cerebral } \\
\text { glucose metabolism reduced } \\
\text { by } 33 \% \text { with a corresponding } \\
\text { reduction in oxygen use for } \\
\text { glucose metabolism from } 97 \% \\
\text { to } 74 \% \text { during } \\
\text { hyperketonaemia. }\end{array}$ & $\begin{array}{l}0.31 \pm 0.17 \mathrm{mM} \text { to } \\
2.16 \pm 0.42 \mathrm{mM} \\
\text { post-infusion }\end{array}$ & Nil noted \\
\hline 2001 & Pan et al. (16) & Case-series & Healthy & 6 & $\begin{array}{l}\text { Dextro-BHB } \\
200 \mathrm{mM} ; \mathrm{pH} \\
7.1\end{array}$ & Fasting & $\begin{array}{l}80 \\
\mu \mathrm{mol} / \mathrm{kg} / \mathrm{min} \\
\text { followed by } \\
20 \\
\mu \mathrm{mol} / \mathrm{kg} / \mathrm{min} \\
\text { for } 75 \mathrm{~min}\end{array}$ & & No & $\begin{array}{l}\text { Cerebral ketone uptake may } \\
\text { be increased during a fasting } \\
\text { state. }\end{array}$ & $\begin{array}{l}0.20 \pm 0.10 \mathrm{mM} \text { to } \\
2.12 \pm 0.30 \mathrm{mM} \\
\text { post-infusion }\end{array}$ & Nil noted \\
\hline 2002 & Pan et al. (17) & Case-series & Healthy & 4 & $\begin{array}{l}\text { Dextro-BHB } \\
200 \mathrm{mM} ; \mathrm{pH} \\
7.1\end{array}$ & Fasting & $\begin{array}{l}16.7 \mathrm{~mL} / \mathrm{min} \\
\text { for } 20 \mathrm{~min} \\
\text { followed by } \\
22 \\
\mu \mathrm{mol} / \mathrm{kg} / \mathrm{min} \\
\text { for } 120 \mathrm{~min}\end{array}$ & & No & $\begin{array}{l}\text { Rapid entry of BHB into the } \\
\text { brain with a distribution similar } \\
\text { to glucose. }\end{array}$ & $\begin{array}{l}\text { Plasma post infusion: } \\
2.25 \pm 0.24 \mathrm{mM} \text {. } \\
\text { Brain post infusion: } \\
0.18 \pm 0.06 \mathrm{mM} \text {. }\end{array}$ & Nil noted \\
\hline 2002 & $\begin{array}{l}\text { Blomqvist } \\
\text { et al. (18) }\end{array}$ & $\begin{array}{l}\text { Placebo- } \\
\text { controlled } \\
\text { single-blinded }\end{array}$ & $\begin{array}{l}\text { T1DM + } \\
\text { Healthy } \\
\text { controls }\end{array}$ & $\begin{array}{l}6 \mathrm{~T} 1 \mathrm{DM} / 6 \\
\text { Controls }\end{array}$ & $\begin{array}{l}\text { Racaemic } \\
\text { BHB }\end{array}$ & Fasting & $\begin{array}{l}6 \mathrm{mg} / \mathrm{kg} / \mathrm{min} \\
\text { over } 20 \text { min } \\
\text { followed by } 3 \\
\mathrm{mg} / \mathrm{kg} / \mathrm{min} \\
\text { continuous } \\
\text { infusion for up } \\
\text { to } 70 \text { min }\end{array}$ & & $\begin{array}{l}\text { For T1DM patients } \\
\text { only: maintain BGL } \\
\text { between } 6 \text { and } 12 \\
\text { mmol/L. }\end{array}$ & $\begin{array}{l}\text { No difference in uptake rate } \\
\text { was observed between T1DM } \\
\text { and Healthy controls. } \\
\text { Rate-limiting step for cerebral } \\
\text { ketone metabolism is transfer } \\
\text { from the blood to brain. }\end{array}$ & $\begin{array}{l}\text { Post-infusion: T1DM } \\
1.28 \pm 0.31 \\
\mu \mathrm{mol} / \mathrm{mL} ; \text { Healthy } \\
0.98 \pm 0.33 \\
\mu \mathrm{mol} / \mathrm{mL}\end{array}$ & Nil noted \\
\hline 2018 & $\begin{array}{l}\text { Svart et al. } \\
\text { (19) }\end{array}$ & $\begin{array}{l}\text { Randomized } \\
\text { placebo- } \\
\text { controlled } \\
\text { crossover }\end{array}$ & Healthy & 9 & $\begin{array}{l}\text { Racaemic } \\
\text { Na-3-OHB } 75 \\
\text { g/L }\end{array}$ & Fasting & $\begin{array}{l}0.22 \mathrm{~g} / \mathrm{kg} / \mathrm{h} \\
3-\mathrm{BHB} \text { for up } \\
\text { to } 4 \mathrm{~h}\end{array}$ & & No & $\begin{array}{l}\text { Cerebral blood flow increased } \\
\text { by } 30 \% \text { with a } 14 \% \text { reduction } \\
\text { in cerebral glucose utilization. } \\
\text { Oxygen consumption } \\
\text { remained unchanged. }\end{array}$ & $\begin{array}{l}0.2 \pm 0.02 \mathrm{mM} \text { to } 5.5 \\
\pm 0.4 \mathrm{mM} \\
\text { post-infusion }\end{array}$ & Nil noted \\
\hline 2020 & $\begin{array}{l}\text { Jensen et al. } \\
\text { (20) }\end{array}$ & $\begin{array}{l}\text { Randomized } \\
\text { placebo- } \\
\text { controlled } \\
\text { double blinded } \\
\text { crossover }\end{array}$ & T2DM & 18 & $\begin{array}{l}\text { Racaemic } \\
\text { Na-3-OHB } \\
7.5 \%\end{array}$ & Fasting & $\begin{array}{l}0.22 \mathrm{~g} / \mathrm{kg} / \mathrm{h} \\
\text { for up to } 3 \mathrm{~h}\end{array}$ & & $\begin{array}{l}0.2 \text { units } / \mathrm{m}^{2} / \mathrm{min} \\
\text { bolus over } 3 \mathrm{~min} \\
\text { followed by } 0.075 \\
\text { units } / \mathrm{m}^{2} / \mathrm{min} \\
\text { continuous infusion }\end{array}$ & $\begin{array}{l}\text { Improved working memory } \\
\text { performance in T2DM patients. } \\
\text { No difference in global } \\
\text { cognitive composite outcome } \\
\text { was observed. }\end{array}$ & $\begin{array}{l}0.1 \pm 0.0 \mathrm{mM} \text { to } 2.4 \\
\pm 0.6 \mathrm{mM} \\
\text { post-infusion }\end{array}$ & $\begin{array}{l}\text { Mild } \\
\text { headache } \\
\text { and light- } \\
\text { headedness }\end{array}$ \\
\hline
\end{tabular}

$A D R$, adverse drug reaction; $B H B$, beta-hydroxybutyrate; $C l$, continuous infusion; $C O$, cardiac output; $E G$, euglycaemic clamp; FFA, free fatty acids; NEFA, non-esterified fatty acids; GFR, glomerular filtration rate; GH, growth hormone; HFrEF, heart failure with reduced ejection fraction; HR, heart rate; NYHA New York Heart Association; SVR, systemic vascular resistance; T1DM, type 1 or insulin-dependent Diabetes mellitus; T2DM, type 2 or non-insulin-dependent Diabetes mellitus; VT, ventricular tachycardia.

Data presented as mean \pm standard error. 
that $\mathrm{BHB}$ administration produces a dose-dependent increase in cardiac output (which are unlikely to be related to myocardial free fatty acid uptake) and that cardiac effects can be seen at physiological concentrations. BHB appeared safe with only two episodes of nausea and one of asymptomatic ventricular tachycardia (known condition) (12).

\section{Ketones and the Brain}

All reviewed studies examined the impact of intravenous $\mathrm{BHB}$ on a variety of clinical and physiological endpoints (Table 2). No publications could be identified examining intravenous $\mathrm{BHB}$ in individuals with neuropathology.

Blomqvist et al. examined the effect of acute hyperketonemia on the cerebral uptake of ketone bodies in nondiabetic subjects and insulin dependent diabetes mellitus patients (18). For both groups, cerebral utilization rates increased proportionately with plasma concentration of ketone bodies suggesting that the transfer from blood to brain was a rate limiting step in ketone body utilization. In contrast, Pan et al. published two studies using magnetic resonance imaging spectroscopy in healthy participants and demonstrated diffuse central nervous system (CNS) ketone uptake with a similar distribution to glucose within $15 \mathrm{~min}$ of administration, achieving peak mean CNS concentrations of $\sim 0.18$ to $0.24 \mathrm{mmol} / \mathrm{L}(16,17)$. This rapid uptake may, in part, be related to increased cerebral blood flow given that cerebral uptake was linearly related to arterial BHB concentration (Mikkelsen et al.) indicating a first-order process such as passive diffusion (21). Hasselbalch et al. showed increased global cerebral blood flow by 39\% ( $p<0.02)$ (15). Moreover, in the absence of exogenous $\mathrm{BHB}, 97 \%$ of oxygen metabolism was used for oxidation of glucose, whereas during acute hyperketonemia, $74 \%$ of oxygen metabolism was due to glucose and $26 \%$ from ketone body oxidation. The overall rate of oxygen metabolism remained constant. Similar findings were identified by Svart et al. with an increased cerebral blood flow of $30 \%$ and a reduction in glucose oxidation by $\sim 14 \%$ (19). The improvements in cerebral blood flow and altered oxygen metabolism may improve working memory in patients with Type 2 Diabetes mellitus. Jensen et al. administered a racemic formulation of $\mathrm{BHB}$ that was associated with a $17 \%$ improvement in working memory (although there was no change in global cognitive function) (20).

\section{Renal Effects}

The impact of $\mathrm{BHB}$ on renal function has also been examined. Fioretto et al. compared the impact of $\mathrm{BHB}$ infusions on the glomerular filtration rate in 11 insulin dependent diabetes mellitus (IDDM) patients and 11 controls (22). Glomerular filtration increased significantly in the IDDM group only, without a change in $\mathrm{pH}$ or bicarbonate in any group. Conversely, there was no change in glomerular filtration following $\mathrm{BHB}$ administration in healthy males despite similar peak $\mathrm{BHB}$ concentrations (23). Wildenhoff investigated renal handling of ketones in IDDM and normal volunteers by administering BHB $(1 \mathrm{~mol} / \mathrm{L})$ at varying rates and measuring renal clearances (24). They noted that at low filtration rates, BHB was completely reabsorbed, that this increased linearly with increasing filtration rate and that a maximal tubular reabsorption rate could not be demonstrated.

\section{Metabolic Effects}

The majority of studies examining intravenous infusions of BHB were conducted to determine the metabolic effects of ketone infusions in normal and diabetic individuals (Table 3). These studies span a number of decades and vary significantly in both quality and study design.

Several studies examined the impact of IV ketone infusion on protein metabolism. Reduced protein catabolism (as determined by decreased plasma alanine concentrations and reduced leucine oxidation) and increased protein synthesis are consistent findings across studies when a $\mathrm{BHB}$ concentration $>1 \mathrm{mmol} / \mathrm{L}$ is achieved $(26,27,29,34)$. Similar findings have been shown in patients post-surgery, or involved in a trauma, which are usually associated with a negative nitrogen balance indicating muscle and protein catabolism. Crowe et al. identified an increased leucine concentration following BHB infusion in post-operative patients while Hiraide et al. noted a decreased alanine release from muscles; both situations suggesting a nett reduction in protein synthesis $(35,38)$. Conversely, Woods et al. did not identify a difference in nitrogen balance in patients following cholecystectomy (31). Prolonged fasting (weeks) in addition to intravenous $\mathrm{BHB}$ increased $\mathrm{BHB}$ concentrations from a mean of $5.72 \mathrm{mmol} / \mathrm{L}-10.39 \mathrm{mmol} / \mathrm{L}$. Despite a supraphysiological $\mathrm{BHB}$ concentration, all patients had a slight reduction in glucose and alanine concentrations while there was no change in insulin, pyruvate, lactate, or glucagon.

Two investigators have examined the impact of systemic inflammation and sepsis on BHB metabolism. Thomsen et al. investigated the impact of exogenous $\mathrm{BHB}$ and free fatty acid supplementation on protein metabolism following lipopolysaccharide infusion in 10 healthy volunteers (44). Infusions of $\mathrm{BHB}(0.18 \mathrm{~g} / \mathrm{kg} / \mathrm{h})$ decreased protein catabolism but did not affect cytokine production following lipopolysaccharide administration. Beylot et al. investigated the effect of $\mathrm{BHB}$ on protein, lipolysis, and glucose production in a group of septic patients (42). Results noted a decrease in free fatty acid and glycerol concentrations with a minor decrease in glucose endogenous production but no reduction in leucine oxidation.

Amiel examined the effect of ketone infusion on counterregulatory hormones during induced hypoglycaemia in 6 healthy volunteers (placebo cross-over) (37). The glucose concentration threshold stimulating release of counterregulatory hormones was lower when $\mathrm{BHB}$ was administered to achieve a concentration of $\sim 0.5 \mathrm{mmol} / \mathrm{L}$. All hormone responses were reduced including noradrenaline, adrenaline, cortisol and growth hormone. Similarly, Veneman et al. noted a 57\% decrease in counter-regulatory hormone concentrations and autonomic symptoms during insulin induced hypoglycaemia in healthy volunteers at $\mathrm{BHB}$ concentrations of $2.9 \mathrm{mmol} / \mathrm{L}$ (43). Conversely, Frølund et al. in a similar study did not identify any symptom modification from $\mathrm{BHB}$ administration following induced hypoglycaemia (28). Moreover, there was no difference in neuroglycopaenic symptoms, adrenaline, noradrenaline and cortisol release, despite a $\mathrm{BHB}$ concentration of up to $4 \mathrm{mmol} / \mathrm{L}$. 
TABLE 3 | Ketone studies with metabolic outcomes.

\begin{tabular}{|c|c|c|c|c|c|c|c|c|c|c|c|c|}
\hline Year & Author (Ref) & Study & Population & $\begin{array}{l}\text { No. } \\
\text { participants }\end{array}$ & Formulation & Fasting & Intervention & $\begin{array}{l}\text { Total average } \\
\text { BHB dose }\end{array}$ & Insulin used & Findings overview & Concentration BHB & ADR \\
\hline 1968 & $\begin{array}{l}\text { Balasse and } \\
\text { Ooms (25) }\end{array}$ & $\begin{array}{l}\text { Placebo- } \\
\text { controlled }\end{array}$ & Healthy & $\begin{array}{l}8 \text { BHB / } 5 \\
\text { Controls }\end{array}$ & $\begin{array}{l}\text { Racaemic } \\
\text { Na-3-OHB }\end{array}$ & Unclear & $\begin{array}{l}5 \mathrm{mmol} / \mathrm{kg} / \mathrm{h} \\
\text { for } 1.5 \mathrm{~h}\end{array}$ & & No & $\begin{array}{l}\text { Ketonaemia up to } 47.6 \\
\mathrm{mg} / 100 \mathrm{~mL} \text { resulted in a } \\
\text { mild alkalosis ( } \mathrm{pH} 7.39 \text { to } \\
7.48 \text { ) with a } 50 \% \text { reduction } \\
\text { in non-esterified fatty acids } \\
\text { and a reduced blood } \\
\text { glucose concentration from } \\
84 \mathrm{mg} / 100 \mathrm{~mL} \text { to } 69 \\
\mathrm{mg} / 100 \mathrm{~mL} \text {, without a } \\
\text { change in insulin } \\
\text { concentration. }\end{array}$ & & $\begin{array}{l}\text { Mild } \\
\text { headache, } \\
\text { thirst }\end{array}$ \\
\hline 1975 & $\begin{array}{l}\text { Sherwin et al. } \\
\text { (26) }\end{array}$ & Case-series & Obese + Healthy & $\begin{array}{l}9 \text { Healthy / } 10 \\
\text { Obese }\end{array}$ & $\begin{array}{l}\text { Racaemic } \\
\text { Na-OHB 40\% }\end{array}$ & $\begin{array}{l}12 \mathrm{~h}, 3 \\
\text { day and } \\
3-5 \\
\text { week } \\
\text { fast }\end{array}$ & $\begin{array}{l}\text { Loading dose } \\
\text { twice } \\
\text { continuous } \\
\text { infusion dose } \\
\text { over } 20 \mathrm{~min} \text {. } \\
\text { Non-obese } \\
\text { controls } 3 \\
\mathrm{mg} / \mathrm{kg} / \mathrm{min} \text { for } \\
3 \mathrm{~h} \text {, Obese } \\
\text { dose } 110 \\
\mathrm{mg} / \mathrm{m}^{2} / \mathrm{min} \\
\text { for } 6 \mathrm{~h} \text {. }\end{array}$ & & & $\begin{array}{l}\text { Healthy group: No change } \\
\text { in insulin, pyruvate, lactate } \\
\text { and glucagon. All patients } \\
\text { exhibited a slight reduction } \\
\text { in both glucose and alanine. }\end{array}$ & $\begin{array}{l}\text { BHB } 0.84 \pm 0.09 \mathrm{mM} \\
\text { after infusion. Basal } \\
\text { obese BHB } 0.12 \pm \\
0.03 \mathrm{mM} \text { increasing to } \\
0.94 \pm 0.10 \mathrm{mM} \\
\text { post- } 6 \mathrm{~h} \text { infusion. } \\
\text { Obese after 3-days } \\
\text { starvation: baseline } \\
\text { BHB } 1.39 \pm 0.22 \mathrm{mM} \\
\text { doubled post infusion. } \\
\text { Obese after } 3-5 \text { week } \\
\text { fast baseline } 5.72 \pm \\
0.64 \mathrm{mM} \text { to peak } 10.39 \\
\pm 0.91 \mathrm{mM} \text {. }\end{array}$ & Nil noted \\
\hline 1976 & $\begin{array}{l}\text { Wildenhoff } \\
\text { (24) }\end{array}$ & Crossover & $\begin{array}{l}\mathrm{T} 1 \mathrm{D}+\text { Healthy } \\
\text { controls }\end{array}$ & $\begin{array}{l}26 \text { T1DM / } 9 \\
\text { Controls }\end{array}$ & $\begin{array}{l}\text { Racaemic } \\
\mathrm{Na-3-OHB} \\
1 \mathrm{M}\end{array}$ & Fasting & $\begin{array}{l}50 \mathrm{mmol} \text { over } \\
4 \mathrm{~min}\end{array}$ & & $\begin{array}{l}\text { Administered to } \\
\text { T1DM, no doses } \\
\text { specified. }\end{array}$ & $\begin{array}{l}\text { Increased urinary excretion } \\
\text { T1DM, normalized with } \\
\text { insulin administration. Lower } \\
\text { ketone elimination } \\
\text { non-obese T2DM patients } \\
\text { but not obese T2DM } \\
\text { patients when compared } \\
\text { with healthy controls. } \\
\text { Treatment with } \\
\text { glibenclamide or phenformin } \\
\text { had a decreased the ketone } \\
\text { elimination in the obese, but } \\
\text { not non-obese T2DM } \\
\text { patients compared with } \\
\text { healthy controls. Decreased } \\
\text { tissue ketone uptake in } \\
\text { non-obese T2DM patients } \\
\text { may mediate lower } \\
\text { elimination. }\end{array}$ & & Nil noted \\
\hline
\end{tabular}


TABLE 3 | Continued

\begin{tabular}{|c|c|c|c|c|c|c|c|c|c|c|c|c|}
\hline Year & Author (Ref) & Study & Population & $\begin{array}{l}\text { No. } \\
\text { participants }\end{array}$ & Formulation & Fasting & Intervention & $\begin{array}{l}\text { Total average } \\
\text { BHB dose }\end{array}$ & Insulin used & Findings overview & Concentration BHB & ADR \\
\hline 1976 & $\begin{array}{l}\text { Sherwin et al. } \\
\text { (27) }\end{array}$ & Crossover & T1DM + Healthy & $\begin{array}{l}7 \text { T1DM / } 12 \\
\text { Healthy }\end{array}$ & $\begin{array}{l}\text { Racaemic } \\
\text { Na-BHB }\end{array}$ & Fasting & $\begin{array}{l}6 \mathrm{mg} / \mathrm{kg} / \mathrm{min} \\
\text { bolus over } \\
20 \mathrm{~min} \\
\text { followed by } 3 \\
\mathrm{mg} / \mathrm{kg} / \mathrm{min} \\
\text { over } 3-4.5 \mathrm{~h} \text {. }\end{array}$ & & $\begin{array}{l}1 \text { participant } \\
1 \mathrm{mU} / \mathrm{kg} / \mathrm{min}\end{array}$ & $\begin{array}{l}\text { Ketone clearance } \\
\text { decreased by } 42 \% \text { in } \\
\text { T1DM. Blood glucose } \\
\text { concentration reduced by } \\
25 \% \text { in T1DM and } \\
\text { marginally reduced in } \\
\text { healthy controls. Alanine } \\
\text { concentration reduced. }\end{array}$ & $\begin{array}{l}\text { T1DM BHB } 0.40 \pm \\
0.08 \mathrm{mM} \text { to } 1.67 \pm \\
0.11 \mathrm{mM} \text {. T1DM AcAc } \\
0.11 \pm 0.02 \mathrm{mM} \text { to } \\
0.41 \pm 0.06 \mathrm{mM} \text {. } \\
\text { Healthy BHB } 0.12 \pm \\
0.020 .82 \pm 0.06 \mathrm{mM} \text {. } \\
\text { Healthy AcAc } 0.04 \pm \\
0.01 \text { to } 0.24 \pm \\
0.01 \mathrm{mM} \text {. }\end{array}$ & Nil noted \\
\hline 1980 & $\begin{array}{l}\text { Frolund et al. } \\
\text { (28) }\end{array}$ & Crossover & Healthy & 6 & $\begin{array}{l}\text { Racaemic } \\
\mathrm{Na}-3-\mathrm{OHB}\end{array}$ & Fasting & $\begin{array}{l}8 \mathrm{mg} / \mathrm{kg} \\
\text { loading dose } \\
\text { followed by } \\
\text { infusion } 4 \\
\mathrm{mg} / \mathrm{kg} / \mathrm{min} \text { for } \\
90 \mathrm{~min} .3 \\
\text { subjects also } \\
\text { had } 26 \mathrm{mg} / \mathrm{kg} \\
\text { loading dose } \\
\text { followed by } \\
13 \mathrm{mg} / \mathrm{kg} / \mathrm{min} \\
\mathrm{Cl}\end{array}$ & $\begin{array}{l}\text { Loading dose } \\
24.20 \mathrm{~g} \text { and } \\
\text { maintenance } \\
\text { dose } 78.94 \mathrm{~g}\end{array}$ & $\begin{array}{l}\text { Insulin } 0.15 \\
\text { units/kg to induce } \\
\text { hypoglycaemia. }\end{array}$ & $\begin{array}{l}\text { Ketone infusion did not } \\
\text { change hypoglycaemic } \\
\text { symptoms. Ketone } \\
\text { administration did not } \\
\text { change catecholamine or } \\
\text { cortisol response to } \\
\text { hypoglycaemia. }\end{array}$ & $\begin{array}{l}0.1 \mathrm{mM} \text { to } 0.45 \mathrm{mM} \text { at } \\
\text { a } 4 \mathrm{mg} / \mathrm{kg} / \mathrm{min} \mathrm{BHB} \\
\text { dose and } 4.0 \mathrm{mM} \\
\text { following a } 13 \\
\mathrm{mg} / \mathrm{kg} / \mathrm{min} \mathrm{BHB} \text { dose. }\end{array}$ & $\begin{array}{l}\text { Shivering } \\
\text { and nausea } \\
\text { in all } \\
\text { patients } \\
\text { receiving } 13 \\
\mathrm{mg} / \mathrm{kg} / \mathrm{min} \\
\text { (high dose) } \\
\text { Na-3-OHB } \\
\text { following } \\
\text { insulin } \\
\text { injection. }\end{array}$ \\
\hline 1983 & $\begin{array}{l}\text { Miles et al. } \\
\text { (29) }\end{array}$ & Crossover & Healthy & 6 & $\begin{array}{l}\text { Racaemic } \\
\mathrm{Na}-\mathrm{OHB} 3 \mathrm{M}, \\
\mathrm{pH} 7\end{array}$ & Fasting & $\begin{array}{l}30 \\
\mu \mathrm{mol} / \mathrm{kg} / \mathrm{min} \\
\text { for } 20 \mathrm{~min} \\
\text { followed by } \\
\mu \mathrm{mol} / \mathrm{kg} / \mathrm{min} \\
\text { for } 3 \mathrm{~h} \text {. Doses } \\
\text { based on } \\
\text { D-enantiomer. }\end{array}$ & & No & $\begin{array}{l}\text { No decrease in proteolysis } \\
\text { with reduced alanine and } \\
\text { free fatty acids. }\end{array}$ & $\begin{array}{l}\text { Total ketone body } \\
\text { baseline } 0.21 \pm \\
0.04 \mathrm{mM} \text { to } 2.33 \pm \\
0.48 \mathrm{mM} \text { post infusion }\end{array}$ & Nil noted \\
\hline 1983 & $\begin{array}{l}\text { Quabbe et al. } \\
\text { (30) }\end{array}$ & Crossover & Healthy & 10 & $\begin{array}{l}\text { Racaemic } \\
\mathrm{Na}-3-\mathrm{OHB} \\
7.5 \%\end{array}$ & Fasting & $15 \mathrm{~g} / \mathrm{h}$ for $3 \mathrm{~h}$ & & $\begin{array}{l}\text { Yes - induce } \\
\text { hypoglycaemia }\end{array}$ & $\begin{array}{l}\text { FFA suppressed during BHB } \\
\text { infusion. Growth hormone } \\
\text { increased. Glucagon } \\
\text { increased by } 10-20 \mathrm{pg} / \mathrm{mL} \text {. } \\
\text { Delayed rebound of FFA and } \\
\text { GH following insulin-induced } \\
\text { hypoglycaemia. }\end{array}$ & & Nil noted \\
\hline 1983 & $\begin{array}{l}\text { Woods et al. } \\
\text { (31) }\end{array}$ & $\begin{array}{l}\text { Placebo } \\
\text { controlled }\end{array}$ & $\begin{array}{l}\text { Post-operative } \\
\text { cholecystectomy }\end{array}$ & $\begin{array}{l}17 \mathrm{BHB} / 15 \\
\text { Controls }\end{array}$ & $\begin{array}{l}\text { Racaemic } \\
\text { Na-3-OHB }\end{array}$ & Fasting & $\begin{array}{l}0.75 \mathrm{~g} / \mathrm{h} \text { for } \\
3 \text { days }\end{array}$ & $54 \mathrm{~g}$ & No & $\begin{array}{l}\text { No difference post-operative } \\
\text { mean daily urinary nitrogen } \\
\text { losses. }\end{array}$ & & Nil noted \\
\hline
\end{tabular}


TABLE 3 | Continued

\begin{tabular}{|c|c|c|c|c|c|c|c|c|c|c|c|c|}
\hline Year & Author (Ref) & Study & Population & $\begin{array}{l}\text { No. } \\
\text { participants }\end{array}$ & Formulation $\mathrm{F}$ & Fasting & Intervention & $\begin{array}{l}\text { Total average } \\
\text { BHB dose }\end{array}$ & Insulin used & Findings overview & Concentration BHB & ADR \\
\hline 1986 & $\begin{array}{l}\text { Bratusch- } \\
\text { Marrain et al. } \\
\text { (32) }\end{array}$ & Crossover & Healthy & 9 & $\begin{array}{l}\text { Racaemic- } \\
\text { Na-OHB 4 M; } \\
\mathrm{pH} 7.24\end{array}$ & Fasting & $\begin{array}{l}30 \\
\mu \mathrm{mol} / \mathrm{kg} / \mathrm{min} \\
\text { for } 20 \mathrm{~min} \\
\text { followed by } \\
15 \\
\mu \mathrm{mol} / \mathrm{kg} / \mathrm{min} \\
\text { for } 4 \mathrm{~h}\end{array}$ & & Yes - EG & $\begin{array}{l}\text { Glucose appearance rate } \\
\text { and metabolism were } \\
\text { unchanged during and after } \\
\text { BHB infusion. }\end{array}$ & $\begin{array}{l}109 \pm 31 \mu \mathrm{M} \text { to } 496 \pm \\
81 \mu \mathrm{M} \text { post-infusion }\end{array}$ & Nil noted \\
\hline 1986 & $\begin{array}{l}\text { Christensen } \\
\text { et al. (23) }\end{array}$ & Case-series & Healthy & 7 & $\begin{array}{l}\text { Racaemic } \\
\mathrm{Na}-\mathrm{BHB} 50 \\
\mathrm{~g} / \mathrm{L}\end{array}$ & Fasting & $\begin{array}{l}0.5 \mathrm{~g} / \mathrm{kg} \text { over } \\
1 \mathrm{~h}\end{array}$ & $35.5 \mathrm{~g}$ & No & $\begin{array}{l}\text { No change in urinary } \\
\text { albumin excretion, GFR or } \\
\text { blood pressure. Small } \\
\text { increase } \\
\text { beta-2-microglobuin } \\
\text { excretion. }\end{array}$ & $\begin{array}{l}0.05 \pm 0.05 \mathrm{mM} \\
\text { baseline to } 1.96 \pm \\
0.53 \mathrm{mM}\end{array}$ & Nil noted \\
\hline 1986 & $\begin{array}{l}\text { Desir et al. } \\
\text { (33) }\end{array}$ & Crossover & Healthy & 7 & $\begin{array}{l}\text { Racaemic- } \\
\mathrm{Na-BHB} \\
40 \%\end{array}$ & Fasting & $\begin{array}{l}1 \mathrm{mmol} / \mathrm{kg} \\
\text { over } 20 \mathrm{~min} \\
\text { followed by } \\
0.01 \\
\mathrm{mmol} / \mathrm{kg} / \mathrm{min} \\
\text { for } 160 \mathrm{~min} \text {. }\end{array}$ & & No & $\begin{array}{l}\text { Increased serum and urine } \\
\mathrm{pH} \text {, reduced potassium } \\
(4.18 \pm 0.09 \text { to } 3.58 \pm \\
0.06 \mathrm{mM}), 35 \% \text { increased } \\
\text { urinary ammonia excretion. } \\
\text { GFR unchanged. }\end{array}$ & $\begin{array}{l}53 \pm 9 \mu \mathrm{mol} / \mathrm{L} \text { to } \sim 200 \\
\mu \mathrm{mol} / \mathrm{L} \text { post-infusion }\end{array}$ & Nil noted \\
\hline 1987 & $\begin{array}{l}\text { Fioretto et al. } \\
\text { (22) }\end{array}$ & Crossover & T1DM + Healthy & $\begin{array}{l}11 \text { T1DM / } 11 \\
\text { Controls }\end{array}$ & $\begin{array}{l}\text { Racaemic 3- } \\
\text { hydroxybutyric } \\
\text { acid/Racaemic } \\
\text { Na-3-OHB }\end{array}$ & Fasting & $\begin{array}{l}40 / 30 \\
\mu \mathrm{mol} / \mathrm{kg} / \mathrm{min} \\
\text { for } 2 \mathrm{~h}\end{array}$ & $\begin{array}{l}30 \mu \mathrm{mol} / \mathrm{kg} \\
/ \mathrm{min}: 367.2 \\
\mathrm{mmol}(46.3 \mathrm{~g}) \\
\text { T1DM and } 378 \\
\text { mmol }(47.66 \mathrm{~g}) \\
\text { Healthy; } 40 \\
\mu \mathrm{mol} / \mathrm{kg} / \\
\mathrm{min}: 489.6 \\
\mathrm{mmol}(61.73 \mathrm{~g}) \\
\text { T1DM and } 504 \\
\text { mmol }(63.55 \mathrm{~g}) \\
\text { Healthy }\end{array}$ & Yes - EG & $\begin{array}{l}\text { GFR increased T1DM. No } \\
\text { change pH or bicarbonate. }\end{array}$ & $\begin{array}{l}\text { Following infusion } 40 \\
\mu \mathrm{mol} / \mathrm{kg} / \mathrm{min} \text { healthy } \\
\text { controls total ketone } \\
\text { body concentration of } \\
2.719 \pm 0.163 \mathrm{mM} \text { and } \\
3.089 \pm 0.149 \mathrm{mM} \\
\text { T1DM. Following } \\
\text { infusion of } 30 \\
\mu \mathrm{mol} / \mathrm{kg} / \mathrm{min} \text {, total } \\
\text { ketone body } \\
\text { concentration of } 0.95 \\
\pm 0.17 \mathrm{mM} \text { and } 1.49 \pm \\
0.27 \mathrm{mM} \text { in T1DM. }\end{array}$ & Nil noted \\
\hline 1988 & Nair et al. (34) & Crossover & Healthy & 13 & $\begin{array}{l}\text { Racaemic } \\
\text { Na-BHB; pH } \\
\text { 6.8-7.4 }\end{array}$ & Fasting & $\begin{array}{l}12.5 \\
\mu \mathrm{mol} / \mathrm{kg} / \mathrm{h}\end{array}$ & $\begin{array}{l}452.63 \mathrm{mmol} \\
(57.07 \mathrm{~g})\end{array}$ & No & $\begin{array}{l}\text { Decreased glucose and free } \\
\text { fatty acids with unchanged } \\
\text { c-peptide, glucagon, } \\
\text { noradrenaline and } \\
\text { adrenaline concentrations. } \\
\text { Decreased leucine oxidation } \\
\text { unrelated to a change in } \\
\text { blood pH; thereby likely } \\
\text { promoting protein synthesis. }\end{array}$ & $\begin{array}{l}<0.5 \mathrm{mM} \text { to } \sim 2 \mathrm{mM} \\
\text { post-infusion. }\end{array}$ & Nil noted \\
\hline
\end{tabular}




\begin{tabular}{|c|c|c|c|c|c|c|c|c|c|c|c|c|}
\hline Year & Author (Ref) & Study & Population & $\begin{array}{l}\text { No. } \\
\text { participants }\end{array}$ & Formulation & Fasting & Intervention & $\begin{array}{l}\text { Total average } \\
\text { BHB dose }\end{array}$ & Insulin used & Findings overview & Concentration BHB & ADR \\
\hline 1989 & $\begin{array}{l}\text { Crowe et al. } \\
\text { (35) }\end{array}$ & Case-series & $\begin{array}{l}\text { Post-operative } \\
\text { Total Hip } \\
\text { Replacements }\end{array}$ & 6 & $\begin{array}{l}\text { Racaemic- } \\
\text { Na-BHB }\end{array}$ & Fasting & $\begin{array}{l}6 \mathrm{mg} / \mathrm{kg} / \mathrm{min} \\
\text { over } 20 \mathrm{mins} \\
\text { followed by } 3 \\
\mathrm{mg} / \mathrm{kg} / \mathrm{min} \text { for } \\
2 \mathrm{~h}\end{array}$ & & No & $\begin{array}{l}\text { Leucine concentrations } \\
\text { and rate of appearance } \\
\text { increased following BHB } \\
\text { infusion. Insulin } \\
\text { concentration not affected } \\
\text { following BHB infusion. }\end{array}$ & $\begin{array}{l}\text { Pre-op } 0.06 \pm 0.01 \text { to } \\
0.69 \pm 0.16 \mathrm{mM} \\
\text { post-infusion. Post-op } \\
0.04 \pm 0.01 \text { to } 0.62 \pm \\
0.12 \mathrm{mM} \text { post-infusion. }\end{array}$ & Nil noted \\
\hline 1990 & $\begin{array}{l}\text { Moller et al. } \\
\text { (36) }\end{array}$ & Cross-over & T1DM & 5 & $\begin{array}{l}\text { Racaemic- } \\
\text { Na-OHB; pH } \\
6.5\end{array}$ & Fasting & $\begin{array}{l}1.8 \\
\mathrm{mmol} / \mathrm{kg} / \mathrm{h} \text { for } \\
20 \mathrm{~min} \text { and } \\
0.9 \\
\mathrm{mmol} / \mathrm{kg} / \mathrm{h} \text { for } \\
100 \mathrm{~min}\end{array}$ & & Yes - EG & $\begin{array}{l}\text { NEFA decreased in both } \\
\text { hypo- and hyperglycaemia } \\
\text { conditions. No change in } \\
\text { NEFA clearance. }\end{array}$ & $\begin{array}{l}\text { Pre-infusion: } \\
\text { euglycaemia } 127 \\
\text { (30-435) } \mu \mathrm{mol} / \mathrm{L} \text { and } \\
\text { hyperglycaemia } 137 \\
\text { (20-350) } \mu \mathrm{mol} / \mathrm{L} \text {. } \\
\text { Post-infusion: } \\
\text { euglycaemia } 770 \\
\text { (520-955) } \mu \mathrm{mol} / \mathrm{L} \text { and } \\
\text { hyperglycaemia } 665 \\
\text { (450-910) } \mu \mathrm{mol} / \mathrm{L} \text {. }\end{array}$ & Nil noted \\
\hline 1991 & $\begin{array}{l}\text { Amiel et al. } \\
\text { (37) }\end{array}$ & $\begin{array}{l}\text { Randomized } \\
\text { placebo- } \\
\text { controlled } \\
\text { crossover }\end{array}$ & Healthy & 6 & $\begin{array}{l}\text { Racaemic } \\
\mathrm{Na}-\mathrm{OHB} \\
6.24-7.38 \\
\mathrm{~g} / 100 \mathrm{~mL}\end{array}$ & Fasting & $\begin{array}{l}6 \mathrm{mg} / \mathrm{kg} / \mathrm{min} \\
\text { for } 20 \mathrm{~min} \\
\text { followed by } 3 \\
\mathrm{mg} / \mathrm{kg} / \mathrm{min} \text {. }\end{array}$ & $48.1 \mathrm{~g}$ & Yes - EG & $\begin{array}{l}\text { Following hypoglycaemia, } \\
\text { all hormone peak } \\
\text { concentrations } \\
\text { (noradrenaline, adrenaline, } \\
\text { cortisol and growth } \\
\text { hormone) were decreased } \\
\text { in subjects receiving BHB } \\
\text { compared with controls. }\end{array}$ & $\begin{array}{l}61 \pm 17 \mu \mathrm{mol} / \mathrm{L} \text { to } 580 \\
\pm 69 \mu \mathrm{mol} / \mathrm{L} \\
\text { post-infusion. }\end{array}$ & Nil noted \\
\hline 1991 & $\begin{array}{l}\text { Hiraide et al. } \\
\text { (38) }\end{array}$ & $\begin{array}{l}\text { Placebo- } \\
\text { controlled }\end{array}$ & Blunt trauma & $\begin{array}{l}11 \mathrm{BHB} / 9 \\
\text { Controls }\end{array}$ & $\begin{array}{l}\text { Racaemic } \\
\text { Na-3-OHB } \\
20 \%\end{array}$ & Unclear & $\begin{array}{l}25 \\
\mu \mathrm{mol} / \mathrm{kg} / \mathrm{min} \\
\text { for } 3 \mathrm{~h}\end{array}$ & $\begin{array}{l}247.5 \mathrm{mmol} \\
(31.21 \mathrm{~g})\end{array}$ & No & $\begin{array}{l}\text { Slight decrease in } \\
\text { non-esterified fatty acids } \\
\text { and alanine release. Mild } \\
\text { alkalosis for both sodium } \\
\text { lactate and BHB infusion. }\end{array}$ & $\begin{array}{l}\text { Total ketone body } \\
210.5 \pm 176.0 \mu \mathrm{mol} / \mathrm{L} \\
\text { to } 1519.2 \pm 420.6 \\
\mu \mathrm{mol} / \mathrm{L} .\end{array}$ & Nil noted \\
\hline 1991 & $\begin{array}{l}\text { Walker et al. } \\
\text { (39) }\end{array}$ & $\begin{array}{l}\text { Randomized } \\
\text { crossover }\end{array}$ & Healthy & 7 & $\begin{array}{l}\text { Racaemic } \\
\text { Na-3-OHB } \\
\text { 3M; pH } 7.2\end{array}$ & Fasting & $\begin{array}{l}15 \\
\mu \mathrm{mol} / \mathrm{kg} / \mathrm{min} \\
\text { over } 4 \mathrm{~h}\end{array}$ & & Yes - EG & $\begin{array}{l}\text { Slight decrease and } \\
\text { increase in glucose and } \\
\text { lactate respectively. BHB } \\
\text { did not inhibit insulin } \\
\text { stimulated glucose uptake. }\end{array}$ & $\begin{array}{l}\text { Total ketone body } \\
\text { levels from } 70 \pm 4 \text { to } \\
450 \pm 30 \mu \mathrm{M} \\
\text { post-infusion. }\end{array}$ & Nil noted \\
\hline 1992 & $\begin{array}{l}\text { Beaufrere } \\
\text { et al. (40) }\end{array}$ & $\begin{array}{l}\text { Placebo } \\
\text { controlled }\end{array}$ & Healthy & $\begin{array}{l}6 \mathrm{BHB} / 4 \\
\text { Controls }\end{array}$ & $\begin{array}{l}\text { Dextro-BHB } \\
0.33 \mathrm{~g} / \mathrm{L} ; \mathrm{pH} \\
6.8\end{array}$ & Fasting & $\begin{array}{l}540 \\
\mu \mathrm{mol} / \mathrm{kg} / \mathrm{h} \text { for } \\
5 \mathrm{~h}\end{array}$ & & No & $\begin{array}{l}73 \% \text { reduction FFA. } \\
\text { Unchanged cortisol, insulin } \\
\text { and C-peptide } \\
\text { concentrations. No } \\
\text { significant change leucine } \\
\text { oxidation. }\end{array}$ & $\begin{array}{l}\text { Total ketone body from } \\
180 \pm 60 \text { to } 1647 \pm \\
275 \mu \mathrm{M}\end{array}$ & Nil noted \\
\hline 1993 & $\begin{array}{l}\text { Chiolero et al. } \\
\text { (41) }\end{array}$ & Crossover & Healthy & 6 & $\begin{array}{l}\text { Racaemic- } \\
\text { Na-OHB }\end{array}$ & Fasting & $\begin{array}{l}20 \\
\mu \mathrm{mol} / \mathrm{kg} / \mathrm{min} \\
\text { for } 3 \mathrm{~h}\end{array}$ & & No & $\begin{array}{l}\text { FFA decreased. Increased } \\
\text { bicarbonate concentration. } \\
\text { Oxygen consumption } \\
\text { increased } 5.5 \% \text {. } \\
\text { Carbohydrate oxidation } \\
\text { inhibited } 25 \% \text {. }\end{array}$ & $\begin{array}{l}0.02 \pm 0.01 \mathrm{mM} \text { to } \\
0.94 \pm 0.07 \mathrm{mM} \\
\text { post-infusion. }\end{array}$ & Nil noted \\
\hline
\end{tabular}


TABLE 3 | Continued

\begin{tabular}{|c|c|c|c|c|c|c|c|c|c|c|c|c|}
\hline Year & Author (Ref) & Study & Population & $\begin{array}{l}\text { No. } \\
\text { participants }\end{array}$ & Formulation & Fasting & Intervention & $\begin{array}{l}\text { Total average } \\
\text { BHB dose }\end{array}$ & Insulin used & Findings overview & Concentration BHB & ADR \\
\hline 1994 & $\begin{array}{l}\text { Beylot et al. } \\
\text { (42) }\end{array}$ & Controlled & $\begin{array}{l}\text { Sepsis patients in } \\
\mathrm{ICU}+\text { Healthy }\end{array}$ & $\begin{array}{l}12 \text { Sepsis/6 } \\
\text { Healthy }\end{array}$ & $\begin{array}{l}\text { Dextro-OHB, } \\
\mathrm{pH} 6.8\end{array}$ & Unclear & $\begin{array}{l}15 \\
\mu \mathrm{mol} / \mathrm{kg} / \mathrm{min} \\
\text { over } 4 \mathrm{~h}\end{array}$ & & No & $\begin{array}{l}\text { No change in pH. Slight } \\
\text { decrease in free fatty acids, } \\
\text { glycerol and endogenous } \\
\text { glucose production. Mild } \\
\text { insulin increase and leucine } \\
\text { oxidation. }\end{array}$ & & Nil noted \\
\hline 1994 & $\begin{array}{l}\text { Veneman } \\
\text { et al. (43) }\end{array}$ & $\begin{array}{l}\text { Randomized, } \\
\text { crossover }\end{array}$ & Healthy & 13 & $\begin{array}{l}\text { Racaemic- } \\
\text { Na-BHB }\end{array}$ & Fasting & $\begin{array}{l}40 \\
\mu \mathrm{mol} / \mathrm{kg} / \mathrm{min} \\
\text { for } 20 \mathrm{~min} \\
\text { followed by } \\
20 \\
\mu \mathrm{mol} / \mathrm{kg} / \mathrm{min}\end{array}$ & & Yes - EG & $\begin{array}{l}\text { BHB infusion decreased } \\
\text { counterregulatory hormone } \\
\text { response to hypoglycaemia } \\
\text { with } 57 \% \text { reduction in } \\
\text { epinephrine and } 28 \% \\
\text { reduction in cortisol. } \\
\text { Reduced neuroglycopenic } \\
\text { symptoms with BHB } \\
\text { infusion. }\end{array}$ & $\begin{array}{l}10 \pm 5 \mu \mathrm{mol} / \mathrm{L} \text { to } 1.9 \\
\mathrm{mmol} / \mathrm{L}\end{array}$ & Nil noted \\
\hline 1997 & $\begin{array}{l}\text { Wildenhoff } \\
\text { (24) }\end{array}$ & Case-series & T1DM + Healthy & $\begin{array}{l}7 \text { T1DM/8 } \\
\text { Healthy }\end{array}$ & $\begin{array}{l}\text { Racaemic- } \\
\text { Na-BHB; } \\
1 \mathrm{M}\end{array}$ & Fasting & $\begin{array}{l}50 \mathrm{mmol} \\
\text { followed by } 0 \\
\text { to } 2.5 \\
\mathrm{mmol} / \mathrm{min}\end{array}$ & & No & $\begin{array}{l}\text { Linear relationship showing } \\
\text { increased total urinary } \\
\text { ketone body excretion and } \\
\text { reabsorption rate with } \\
\text { increasing urinary filtration } \\
\text { rate in both healthy controls } \\
\text { and T1DM patients. }\end{array}$ & $\begin{array}{l}0.015 \text { to } 2.91 \mathrm{mM} \\
\text { post-infusion. }\end{array}$ & Nil noted \\
\hline 2015 & $\begin{array}{l}\text { Mikkelsen } \\
\text { et al. (21) }\end{array}$ & Case-series & Healthy & 6 & Dextro-OHB & Fasting & $\begin{array}{l}4.7 \\
\mu \mathrm{mol} / \mathrm{kg} / \mathrm{min} \\
\text { for } 1 \mathrm{~h} \text { then } \\
9.4 \\
\mu \mathrm{mol} / \mathrm{kg} / \mathrm{min} \\
\text { for } 1 \mathrm{~h} \text { then } \\
18.8 \\
\mu \mathrm{mol} / \mathrm{kg} / \mathrm{min} \\
\text { thereafter for } \\
50 \text { min. }\end{array}$ & $\begin{array}{l}159.89 \mathrm{mmol} \\
(20.16 \mathrm{~g})\end{array}$ & No & $\begin{array}{l}\text { 14\% reduction glucose } \\
\text { appearance and } 37 \% \\
\text { decrease lipolytic rate. } \\
\text { Insulin and glucagon } \\
\text { concentrations unchanged. } \\
\text { Cerebral OHB uptake } \\
\text { kinetics linear, whereas } \\
\text { skeletal muscle kinetics } \\
\text { saturable. }\end{array}$ & Peak $1.7 \mathrm{mM}$ & Nil noted \\
\hline 2018 & $\begin{array}{l}\text { Thomsen } \\
\text { et al. (44) }\end{array}$ & $\begin{array}{l}\text { Double } \\
\text { blinded } \\
\text { placebo- } \\
\text { controlled } \\
\text { crossover }\end{array}$ & Healthy & 10 & $\begin{array}{l}\text { Racaemic } \\
\text { Na-3-OHB } \\
7.5 \%\end{array}$ & Fasting & $\begin{array}{l}0.18 \mathrm{~g} / \mathrm{kg} / \mathrm{h} \\
3-\mathrm{OHB}\end{array}$ & $101.05 \mathrm{~g}$ & Yes - EG & $\begin{array}{l}\text { Net decrease protein loss. } \\
\text { No effect on cytokine } \\
\text { production. }\end{array}$ & $\begin{array}{l}\text { BHB basal } 115(95 \% \\
\text { Cl 20-210) to } 3449 \\
\text { (1249-5652) mM. BHB } \\
\text { clamp 12 (95\% Cl } \\
2-22) \text { to } 3280(95 \% \mathrm{Cl} \\
\text { 1184-5375) mM. }\end{array}$ & Nil noted \\
\hline 2020 & $\begin{array}{l}\text { Lauritzen } \\
\text { et al. (45) }\end{array}$ & $\begin{array}{l}\text { Randomized } \\
\text { placebo- } \\
\text { controlled } \\
\text { crossover }\end{array}$ & Healthy & 9 & $\begin{array}{l}\text { Racaemic- } \\
\text { Na-OHB }\end{array}$ & Fasting & $\begin{array}{l}0.22 \mathrm{~g} / \mathrm{kg} / \mathrm{h} \\
\text { for } 4 \mathrm{~h}\end{array}$ & & No & $\begin{array}{l}\text { Insulin, glucagon and } \\
\text { Fibroblast growth factor-21 } \\
\text { concentrations unchanged. }\end{array}$ & $\begin{array}{l}0.0 \pm 0.0 \mathrm{mM} \text { to } 5.5 \pm \\
0.4 \mathrm{mM}\end{array}$ & Nil noted \\
\hline
\end{tabular}

ADR, adverse drug reaction; BHB, beta-hydroxybutyrate; Cl, continuous infusion; $C O$, cardiac output; $E G$, euglycaemic clamp; FFA, free fatty acids; NEFA, non-esterified fatty acids; GFR, glomerular filtration rate; GH, growth hormone; HFrEF, heart failure with reduced ejection fraction; HR, heart rate; NYHA New York Heart Association; SVR, systemic vascular resistance; T1DM, type 1 or insulin-dependent Diabetes mellitus; T2DM, type 2 or non-insulin-dependent Diabetes mellitus; VT, ventricular tachycardia.

Data presented as mean \pm standard error. 
The effect of BHB appears independent of insulin stimulated glucose uptake (39). Balasse et al. evaluated the role of insulin in modifications of blood glucose and non-esterified fatty acids induced by BHB (25). $\beta$-hydroxybutyrate decreased nonesterified fatty acids by $50 \%$ while the insulin concentration remained unchanged. Similarly, Lauritzen et al. investigated the effect of acute hyperketonemia on the uptake of glucose and palmitate in abdominal organs, adipose tissue, and skeletal muscle (45). $\beta$-hydroxybutyrate infusion did not affect glucose or palmitate uptake suggesting that ketone bodies are selectively used by critical organs such as heart and brain.

\section{STUDIES WITH ACETOACETATE}

Although most investigators have utilized $\mathrm{BHB}$, acetoacetate has also been studied. Nosadini et al. investigated the kidney haemodynamics after ketone body and amino acid infusions in IDDM (46). Fifteen patients with IDDM and eight controls received an infusion of acetoacetate at $25 \mathrm{mmol} / \mathrm{kg} / \mathrm{min}$ for $3 \mathrm{~h}$. The total ketone bodies reached $2.2 \pm 1.5 \mathrm{mmol} / \mathrm{l}$ and was similar between groups. While glomerular filtration increased, albumin excretion in IDDM patients did not. Similarly, Owen et al. administered acetoacetate to assess influence of ketone bodies on insulin and free fatty acid release and to determine rates of uptake and distribution (47). Total ketone bodies reached $4.74 \pm 0.39 \mathrm{mmol} / \mathrm{l}$. Tissue utilization rates increased with $\mathrm{BHB}$ concentration to a peak at $4-5 \mathrm{mmol} / \mathrm{l}$. Free fatty acid decreased, and glucose was unchanged. Frey et al. administered acetoacetate to eight normal subjects at $1.9 \mathrm{mmol} / \mathrm{min} \times 1.73 \mathrm{~m}^{2}$ for $210 \mathrm{~min}$ and evaluated changes during exercise (48). Acetoacetate peaked at $2.54 \pm 0.16 \mathrm{mmol} / \mathrm{l}$. Free fatty acid and glucose declined as did ketones during exercise.

\section{BHB Dose, Pharmacokinetics and Adverse Events}

Of studies examined, only eight provided average patient weight allowing for estimation of total ketone infused during study period. Total BHB administered ranged from $\sim 30-101 \mathrm{~g}$ (in the study by Thomsen et al.) (44). The racemic isomer was utilized in most studies. Most authors reported on BHB and acetylacetate concentrations rather than total dose. Furthermore, plasma concentrations of $\mathrm{BHB}$ ranged from $<1 \mathrm{mmol} / \mathrm{l}$ to $>5 \mathrm{mmol} / \mathrm{l}$ with the highest $10.39 \pm 0.91 \mathrm{mmol} / \mathrm{l}$ recorded in the study by Sherwin et al. in fasted patients (27). Many studies demonstrated a rapid increase in plasma $\mathrm{BHB}$ with some reaching concentrations of $>1 \mathrm{mmol} / \mathrm{l}$ within $15 \mathrm{~min}$. Sherwin et al. investigated the effect of diabetes mellitus on ketone body removal rates, comparing 12 healthy participants with seven IDDM volunteers (27). Baseline ketone body concentrations were higher in IDDM then controls with an approximately twofold higher BHB peak concentration in patients with IDDM. Clearance of ketones for IDDM was $42 \%$ below healthy participants. They also noted a $25 \%$ decrease in glucose and ketone disposal in IDDM.

A common concern of exogenous ketone supplementation is the risk of ketoacidosis, especially in diabetics $(49,50)$. There are no reports in the literature of diabetic ketoacidosis (DKA) resulting from intravenous ketone supplementation (although some trials excluded diabetic patients) which appears to improve glucose control in the short term. Furthermore, data from trials using enteral ketone supplementation to induce ketosis suggest the incidence of DKA is exceedingly low and limited to case reports. Bolla et al. reviewed the literature pertaining to ketogenic diets in diabetics, both type 1 and 2, and suggests that ketogenic diets were safe although close monitoring for DKA was necessary (51). Case reports from children with diabetes and epilepsy managed with a ketogenic diet suggest the treatment was safe provided patients are closely monitored. The few case reports of patients developing DKA secondary to ketogenic diets appears to be limited to individuals with undiagnosed type 1 IDDM who initiated the ketogenic diets and subsequently developed DKA (52-54).

Overall, it appears that the ketone infusions were well tolerated with minimal reported side effects. Common reported issues included a tendency toward alkalemia secondary to an increase in bicarbonate (although few reported $\mathrm{pH}>7.50$ ), glucose concentrations tended to decrease although remained within the normal range, protein in the form of alanine decreased and lactate increased (but remained in the normal range). BHB appears to be readily taken up by the brain and leads to increase blood flow including cerebral, renal, and cardiac. Human studies are consistent with animal studies which demonstrated significant increase in cardiac function with $\mathrm{BHB}$ infusions. Overall, intravenous ketone administration appears safe and well tolerated.

\section{DISCUSSION}

Our review has highlighted that the intravenous administration of $\mathrm{BHB}$ is able to rapidly achieve supraphysiological BHB concentrations and appears safe, although the total number of study participants remains small. Most physiological effects appear to occur at systemic BHB concentrations exceeding $2 \mathrm{mmol} / \mathrm{L}$, which is achievable after a dose of $\sim 0.2 \mathrm{~g} / \mathrm{kg} / \mathrm{h}$. However, no pharmacokinetic study has been performed in patients receiving intravenous $\mathrm{BHB}$.

\section{Why Consider an Intravenous Formulation?}

Until recently, generating significant ketosis in adults was difficult and required either prolonged fasting or a ketogenic diet. As the increase in plasma ketones via these techniques could take several days, neither option was useful in either the sub-acute or acute setting where a rapid increase in ketones was required (eg. Status epilepticus, acute brain injury, cardiac failure etc.). Given the encouraging evidence, both theoretical and experimental, that ketone supplementation may improve cerebral energetics post-acute brain injury, an IV formulation could likely provide a means of rapidly and predictably increasing plasma ketone concentrations. In $2005 \mathrm{a}$ review paper was published examining an intravenous ketone formulation containing the sodium salt of dextro-BHB (KTX 0101) produced by a company called KetoCytonyx (55). The company claimed to have undertaken 
a Phase I trial where KTX 0101 was administered to 20 healthy volunteers in whom it was reportedly well tolerated with no evidence of serious side effects. The intention of KetoCytonyx was to administer their formulation to patients undergoing cardiopulmonary bypass to preserve the metabolic function of neurons during transient ischemia caused by microemboli generated during the procedure in the hope of reducing adverse neurological and psychiatric outcomes. However, the study was never published in a peer review journal and no further development of the product has taken place.

Intravenous administration should theoretically increase both plasma and cerebral ketone concentrations, with minimal adverse reactions. This was demonstrated by Neilsen et al. who were able to increase plasma BHB concentrations to above $3 \mathrm{mmol} / \mathrm{l}$ within $3 \mathrm{~h}$ of initiating an infusion of a $7.5 \% \mathrm{Na}-3-\mathrm{OHB}$ solution (14). This was also demonstrated by Pan et al. who noted an increase in plasma $\mathrm{BHB}$ from $0.20 \pm 0.10 \mathrm{mmol} / \mathrm{l}$ to $2.12 \pm$ $0.30 \mathrm{mmol} / \mathrm{l}$ and cerebral tissue BHB from $0.16 \pm 0.07 \mathrm{mmol} / \mathrm{l}$ to $0.24 \pm 0.04 \mathrm{mmol} / \mathrm{l}$ following a $75 \mathrm{~min}$ infusion of dextroBHB $(16,17)$. This has also been demonstrated in animal models where a $6 \mathrm{~h}$ infusion of hypertonic solution containing 120 $\mathrm{mmol} / \mathrm{L}$ BHB increased plasma BHB from $0.26 \pm 0.05 \mathrm{mmol} / \mathrm{l}$ to $0.70 \pm 0.27 \mathrm{mmol} / \mathrm{l}$ with a proportional increase in cerebral tissue $\mathrm{BHB}$, which was related to the concentration of $\mathrm{BHB}$ in the solution.

The study by Neilsen et al. is one of the only studies examining ketone supplementation in a disease state in humans (14). Intravenous $\mathrm{BHB}$ was demonstrated to significantly improve the ejection fraction in patients with heart failure in a dose-dependent manner. This was the most recent study to demonstrate a clear clinical benefit for intravenous ketone administration and the first to demonstrate that modulation of circulating ketone levels may represent a novel treatment principle in patients with heart failure. Importantly, these benefits were noted with minimal side effects suggesting intravenous $\mathrm{BHB}$ is safe, at least in the short term. However, few studies have investigated prolonged administration of exogenous intravenous ketones.

Although ketone infusions modulate several physiological endpoints, little is known about their impact in disease states. Certainly, there is more data from enterally induced ketosis which suggests that supplementation is effective and beneficial, but ketosis secondary to intravenous administration needs further research. Also, unlike oral supplementation which has been studied over days and weeks, the longest duration reported for intravenous ketones was by Woods et al. who administered BHB for 3 days (31). Most other reports use infusions of less than $6 \mathrm{~h}$. This is significant as long-term infusion may lead to an increased $\mathrm{pH}$. This would depend on a number of factors such as the $\mathrm{BHB}$ concentration in solution and total dose administered. Neilsen et al. noted an increase in plasma $\mathrm{pH}$ in the ketone (7.46 $\pm 0.03)$ compared with saline $(7.42 \pm 0.06)$ groups $(p=0.001)$ and here the infusion was only for $3 \mathrm{~h}$. Further study is necessary to examine $\mathrm{pH}$ changes following longer infusions.

\section{Why Hasn't an Intravenous BHB Formulation Been Developed?}

There are several reasons why an intravenous formulation has yet to be commercially developed. First, there is a paucity of data regarding ketone pharmacokinetics and pharmacodynamics. The complexity is increased given that there are two stochiometric formulations, the dextro- and levo- isomers. Most prior studies have made their formulation utilizing a racemic mixture of $\mathrm{BHB}$ (containing a 50:50 mixture of dextro and levo isomers). There is some evidence that levo-BHB is not metabolized significantly into energy intermediates and is slowly excreted in the urine, therefore a pure dextro-BHB containing formulation would likely be the most effective. Moreover, the specific target concentration for optimal efficacy in disease states is unknown. Early reports by Cahill et al. based on physiological studies suggested that a plasma concentration of $>4 \mathrm{mmol} / \mathrm{l}$ was required to provide adequate cerebral levels based on evidence from starvation rather than intervention studies (56). More recently this has been questioned as the correlation between plasma concentration and efficacy is poor. The majority of clinical research comes from the pediatric epilepsy population where data suggests that plasma concentration does not correlate with efficacy and often anti-convulsant effects continue long after ketogenic diet is ceased (57). Therefore, the optimal dose and formulation $\mathrm{BHB}$ concentration is unknown, although a potential starting concentration may be to target $>2 \mathrm{mmol} / \mathrm{L}$.

Second, the optimal formulation is unknown. Prior studies have included a large variety of ketone concentrations of different solutions. Nielsen et al. utilized a $7.5 \%$ racemic solution, but the infusions ran for a maximum of $3 \mathrm{~h}$ and as previously noted; it is likely that this solution would be alkalinizing if infused for prolonged periods (14). A $70 \mathrm{~kg}$ patient would have received $\sim 38 \mathrm{~g}$ of $\mathrm{BHB}$ over the $3 \mathrm{~h}$ period (although authors did not report on weight). This is in contrast with the approximate $49 \mathrm{~g}$ received by participants in the Clarke et al. study for a patient of average weight (58). Animal studies suggest administration of $<1 \mathrm{~g} / \mathrm{kg}$ per day is safe. Potential side effects include a dose dependent alkalosis, hypernatraemia and fluid overload.

Third, the cost of producing an intravenous ketone formulation using commercially available $\mathrm{BHB}$ salts is prohibitively high, particularly with the high doses of $\mathrm{BHB}$ that may be required $(>0.2 \mathrm{~g} / \mathrm{kg} / \mathrm{h})$. The cost is further increased if a specific enantiomer (dextro-BHB) is desired. Lastly, given the high cost of producing intravenous ketone formulations, there is a paucity of published literature in disease states that has likely deterred appropriate investment in this potential drug treatment. Less expensive production methods are possible by hydrolysis of the poly-D-BHB obtained by fermentation of Alcaligenes eutrophus although the regulatory hurdles would likely be restrictive. Another option would be to produce an intravenous formulation using the ketone ester. There are however concerns that this may not be effective as part of the metabolism of the ketone ester takes place in the gut. Derochers et al. demonstrated that an intravenous formulation of $(R$, S)-1,3-butanediol acetoacetate ester given parenterally to pigs, 
produced total ketone levels of $5 \mathrm{mmol} / \mathrm{L}$ without deleterious side effects (59). No studies in humans exist.

Taken together, there is a compelling argument for the development of an intravenous ketone formulation. Certainly, there is enough research to suggest that intravenous delivery of ketones is safe for short treatment durations and leads to a rapid increase in plasma ketone concentrations. Potential advantages of an intravenous formulation include the ability to administer to sedated patients and those with swallowing abnormalities, as well as dose titration to the desired plasma concentration. Currently the cost of producing an intravenous formulation is restrictive but as noted, other sources of BHB could be utilized to reduce costs of production. The production of a commercially available product could potentially lead to a rapid increase in research and potential applications of this useful energy substrate.

\section{Are There Enteral Alternatives?}

While generally effective at inducing ketosis over time, the ketogenic diet and other traditional enteral ketogenic formulations may have limited utility in the setting of acute neurological disorders such as stroke or traumatic brain injury. Recently, the development of oral ketone esters, currently marketed as HMVN or KetoneAid, has led to the potential to increase ketones rapidly via the enteral route. Pharmacokinetic studies in healthy volunteers of ketone esters by Clarke et al. demonstrated peaks of $3.30 \mathrm{mmol} / \mathrm{l}$ at $2.5 \mathrm{~h}$ post ingestion (58). This is comparable to the peak concentrations noted by Neilsen et al. following intravenous administration (14). Interestingly, the oral ketone ester was able to reach similar concentrations within a similar time scale but was associated with significant gastrointestinal side-effects. Although subjects were fasted for $10 \mathrm{~h}$ prior to the study, evidence suggests that ketone ester supplementation leads to increased $\mathrm{BHB}$ concentrations even in the non-fasted state. The oral ketone ester supplements are registered as food, not drugs, and marketed as a performance enhancer for athletes. As such, there are no clinical trials in humans with cerebral or cardiac pathology. The only human study published to date is a case report on a patient with severe

\section{REFERENCES}

1. Randle PJ, Newsholme EA, Garland PB. Regulation of glucose uptake by muscle. 8 effects of fatty acids, ketone bodies and pyruvate, and of alloxan-diabetes and starvation, on the uptake and metabolic fate of glucose in rat heart and diaphragm muscles. Biochem J. (1964) 93:65265. doi: 10.1042/bj0930652

2. François B, Bachmann C, Schutgens RBH. Glucose metabolism in a child with 3-hydroxy-3-methylglutaryl-coenzyme a lyase deficiency. J Inherit Metab Dis. (1981) 4:163-4. doi: 10.1007/BF02263641

3. Yassen KA, Galley HF, Lee A, Webster NR. Mitochondrial redox state in the critically ill. Br J Anaesth. (1999) 83:325-7. doi: 10.1093/bja/83.2.325

4. Iimuro Y, Yamamoto M, Inoue S, Kohno H, Matsumoto Y. Superoxide production by liver macrophages in a septic rat model-relation to arterial ketone body ratio. Eur Surg Res. (1992) 24:363-71. doi: 10.1159/00012 9229

5. Levy B, Sadoune LO, Gelot AM, Bollaert PE, Nabet P, Larcan A. Evolution of lactate/pyruvate and arterial ketone body ratios in the early course of catecholamine-treated septic shock. Crit
Alzheimer's Disease whose cognitive function was significantly improved by the administration of a ketone monoester, $28.7 \mathrm{~g}$ thrice-daily for 20 months. This was well tolerated with no severe adverse reactions.

\section{CONCLUSION}

Intravenously administered $\mathrm{BHB}$ can rapidly increase $\mathrm{BHB}$ concentrations to $>2 \mathrm{mmol} / \mathrm{L}$, while only minimally reducing glucose concentrations. Overall, intravenous BHB appears safe. Further research is required to determine the likely optimal dose and future clinical studies to ascertain the potential benefit of $\mathrm{BHB}$ in specific disease states such as neurological conditions.

\section{DATA AVAILABILITY STATEMENT}

The original contributions presented in the study are included in the article/Supplementary Material, further inquiries can be directed to the corresponding author.

\section{AUTHOR CONTRIBUTIONS}

HW conceptualized the review, reviewed articles for inclusion, performed the second review of data, and assisted with manuscript writing. AH designed the search strategy, reviewed articles for inclusion, performed the initial data extraction, and assisted with manuscript writing. SW, AG, and MT assistance with review design and manuscript writing. All authors contributed to the article and approved the submitted version.

\section{SUPPLEMENTARY MATERIAL}

The Supplementary Material for this article can be found online at: https://www.frontiersin.org/articles/10.3389/fmed. 2021.740374/full\#supplementary-material
Care Med. (2000) 28:114-9. doi: 10.1097/00003246-200001000-0 0019

6. Gasior M, Rogawski MA, Hartman AL. Neuroprotective and diseasemodifying effects of the ketogenic diet. Behav Pharmacol. (2006) 17:4319. doi: 10.1097/00008877-200609000-00009

7. Barañano KW, Hartman AL. The ketogenic diet: uses in epilepsy and other neurologic illnesses. Curr Treat Options Neurol. (2008) 10:4109. doi: 10.1007/s11940-008-0043-8

8. Stafstrom CE, Rho JM. The ketogenic diet as a treatment paradigm for diverse neurological disorders. Front Pharmacol. (2012) 3:59. doi: 10.3389/fphar.2012.00059

9. Paoli A, Bianco A, Damiani E, Bosco G. Ketogenic diet in neuromuscular and neurodegenerative diseases. Biomed Res Int. (2014) 2014:474296. doi: 10.1155/2014/474296

10. Paoli A, Grimaldi K, Toniolo L, Canato M, Bianco A, Fratter A. Nutrition and acne: therapeutic potential of ketogenic diets. Skin Pharmacol Physiol. (2012) 25:111-7. doi: 10.1159/000336404

11. Stewart RA, Wallentin L, Benatar J, Danchin N, Hagström E, Held C, et al. Dietary patterns and the risk of major adverse cardiovascular events in a global 
study of high-risk patients with stable coronary heart disease. Eur Heart J. (2016) 37:1993-2001. doi: 10.1093/eurheartj/ehw125

12. Vanoverschelde JL, Wijns W, Kolanowski J, Bol A, Decoster PM, Michel C, et al. Competition between palmitate and ketone bodies as fuels for the heart: study with positron emission tomography. Am J Physiol. (1993) 264:H701-7. doi: 10.1152/ajpheart.1993.264.3.H701

13. Gormsen LC, Svart M, Thomsen HH, Søndergaard E, Vendelbo MH, Christensen $\mathrm{N}$, et al. Ketone body infusion with 3-hydroxybutyrate reduces myocardial glucose uptake and increases blood flow in humans: a positron emission tomography study. J Am Heart Assoc. (2017) 27:e005066. doi: 10.1161/JAHA.116.005066

14. Nielsen R, Moller N, Gormsen LC, Tolbod LP, Hansson NH, Sorensen J, et al. Cardiovascular effects of treatment with the ketone body 3hydroxybutyrate in chronic heart failure patients. Circulation. (2019) 139:2129-41. doi: 10.1161/CIRCULATIONAHA.118.036459

15. Hasselbalch SG, Madsen PL, Hageman LP, Olsen RS, Justesen N, Holm S, et al. Changes in cerebral blood flow and carbohydrate metabolism during acute hyperketonemia. Am J Physiol Endocrinol Metab. (1996) 270:E74651. doi: 10.1152/ajpendo.1996.270.5.E746

16. Pan JW, Telang FW, Lee JH, De Graaf RA, Rothman DL, Stein DT, et al. Measurement of $\beta$-hydroxybutyrate in acute hyperketonemia in human brain. J Neurochem. (2001) 79:539-44. doi: 10.1046/j.1471-4159.2001.00575.x

17. Pan JW, De Graaf RA, Petersen KF, Shulman GI, Hetherington HP, Rothman DL. [2,4-13C2]- $\beta$-hydroxybutyrate metabolism in human brain. J Cereb Blood Flow Metab. (2002) 22:890-8. doi: 10.1097/00004647-200207000-00014

18. Blomqvist G, Alvarsson M, Grill V, Von Heijne G, Ingvar M, Thorell JO, et al. Effect of acute hyperketonemia on the cerebral uptake of ketone bodies in nondiabetic subjects and IDDM patients. Am J Physiol Endocrinol Metab. (2002) 283:E20-8. doi: 10.1152/ajpendo.00294.2001

19. Svart M, Gormsen LC, Hansen J, Zeidler D, Gejl M, Vang K, et al. Regional cerebral effects of ketone body infusion with 3-hydroxybutyrate in humans: reduced glucose uptake, unchanged oxygen consumption and increased blood flow by positron emission tomography. A randomized, controlled trial. PLoS ONE. (2018) 13:e0190556. doi: 10.1371/journal.pone.0190556

20. Jensen NJ, Nilsson M, Ingerslev JS, Olsen DA, Fenger M, Svart M, et al. Effects of $\beta$-hydroxybutyrate on cognition in patients with type 2 diabetes. Eur $J$ Endocrinol. (2020) 182:233-42. doi: 10.1530/EJE-19-0710

21. Mikkelsen KH, Seifert T, Secher NH, Grøndal T, van Hall G. Systemic, cerebral and skeletal muscle ketone body and energy metabolism during acute hyperD-ß-hydroxybutyratemia in post-absorptive healthy males. J Clin Endocrinol Metab. (2015) 100:636-43. doi: 10.1210/jc.2014-2608

22. Fioretto P, Trevisan R, Velussi M, Cernigoi A, De Riva C, Bressan M, et al. Glomerular filtration rate is increased in man by the infusion of both D,L3-hydroxybutyric acid and sodium D,L-3-hydroxybutyrate. J Clin Endocrinol Metab. (1987) 65:331-8. doi: 10.1210/jcem-65-2-331

23. Christensen CK, Schmitz O, Pedersen EB, Alberti KG, Mogensen CE. Effect of 3-hydroxybutyrate infusion on urinary protein excretion in healthy man. Scand J Clin Lab Invest. (1986) 46:239-43. doi: 10.3109/00365518609083665

24. Wildenhoff KE. Blood ketone body disappearance rate in diabetics and normals after rapid infusion of dl-3-hydroxybutyrate. Studies before and after diabetic treatment. Acta Med Scand. (1976) 200:79-86. doi: 10.1111/j.0954-6820.1976.tb08200.x

25. Balasse E, Ooms HA. Changes in the concentrations of glucose, free fatty acids, insulin and ketone bodies in the blood during sodium beta-hydroxybutyrate infusions in man. Diabetologia. (1968) 4:133-5. doi: 10.1007/BF01219433

26. Sherwin RS, Hendler RG, Felig P. Effect of ketone infusions on amino acid and nitrogen metabolism in man. J Clin Invest. (1975) 55:138290. doi: 10.1172/JCI108057

27. Sherwin RS, Hendler RG, Felig P. Effect of diabetes mellitus and insulin on the turnover and metabolic response to ketones in man. Diabetes. (1976) 25:776-84. doi: 10.2337/diab.25.9.776

28. Frølund L, Kehlet H, Christensen NJ, Alberti KG. Effect of ketone body infusion on plasma catecholamine and substrate concentrations during acute hypoglycemia in man. J Clin Endocrinol Metab. (1980) 50:5579. doi: 10.1210/jcem-50-3-557

29. Miles JM, Nissen SL, Rizza RA. Failure of infused $\beta$ hydroxybutyrate to decrease proteolysis in man. Diabetes. (1983) 32:197-205. doi: 10.2337/diab.32.3.197
30. Quabbe HJ, Trompke M, Luyckx AS. Influence of ketone body infusion on plasma growth hormone and glucagon in man. J Clin Endocrinol Metab. (1983) 57:613-8. doi: 10.1210/jcem-57-3-613

31. Woods WGA, Pawan GLS, Semple SJG, Ralphs DNL. Effect of intravenous 3-hydroxybutyrate on postoperative nitrogen balance. Hum Nutr Clin Nutr. (1983) 37:391-4. doi: 10.1016/s0140-6736(83)91560-x

32. Bratusch-Marrain PR, DeFronzo RA. Failure of hyperketonemia to alter basal and insulin-mediated glucose metabolism in man. Horm Metab Res. (1986) 18:185-9. doi: 10.1055/s-2007-1012266

33. Desir G, Bratusch-Marrain P, DeFronzo RA. Effect of hyperketonemia on renal ammonia excretion in man. Metab Clin Exp. (1986) 35:73643. doi: 10.1016/0026-0495(86)90241-6

34. Nair KS, Welle SL, Halliday D, Campbell RG. Effect of $\beta$-hydroxybutyrate on whole-body leucine kinetics and fractional mixed skeletal muscle protein synthesis in humans. J Clin Inves. (1988) 82:198-205. doi: 10.1172/JCI113570

35. Crowe PJ, Royle GT, Wagner D, Burke JF. Does hyperketonemia affect protein or glucose kinetics in postabsorptive or traumatized man? J Surg Res. (1989) 47:313-8. doi: 10.1016/0022-4804(89)90141-8

36. Møller N, Jørgensen JO, Møller J, Orskov L, Pørksen N, Alberti KG, et al. Lack of effects of hyperglycemia on the disposal of 3-hydroxybutyrate in insulin-dependent diabetic patients. Acta Endocrinol. (1990) 123:62932. doi: 10.1530/acta.0.1230629

37. Amiel SA, Archibald HR, Chusney G, Williams AJ, Gale EA. Ketone infusion lowers hormonal responses to hypoglycaemia: evidence for acute cerebral utilization of a non-glucose fuel. Clin Sci. (1991) 81:18994. doi: $10.1042 / \operatorname{cs} 0810189$

38. Hiraide A, Katayama M, Sugimoto H, Yoshioka T, Sugimoto T. Effect of 3-hydroxybutyrate on posttraumatic metabolism in man. Surgery. (1991) 109:176-81.

39. Walker M, Fulcher GR, Marsiaj H, Orskov H, Alberti KGMM. The independent effect of ketone bodies on forearm glucose metabolism in normal man. Scand J Clin Lab Invest. (1991) 51:605-13. doi: 10.1080/00365519109104571

40. Beaufrère B, Chassard D, Broussolle C, Riou JP, Beylot M. Effects of D-beta-hydroxybutyrate and long- and medium-chain triglycerides on leucine metabolism in humans. Am J Physiol. (1992) 262:E26874. doi: 10.1152/ajpendo.1992.262.3.E268

41. Chioléro R, Mavrocordatos P, Burnier P, Cayeux MC, Schindler C, Jéquier E, et al. Effects of infused sodium acetate, sodium lactate, and sodium betahydroxybutyrate on energy expenditure and substrate oxidation rates in lean humans. Am J Clin Nutr. (1993) 58:608-13. doi: 10.1093/ajcn/58.5.608

42. Beylot M, Chassard D, Chambrier C, Guiraud M, Odeon M, Beaufrère B, et al. Metabolic effects of a D-beta-hydroxybutyrate infusion in septic patients: inhibition of lipolysis and glucose production but not leucine oxidation. Crit Care Med. (1994) 22:1091-8. doi: 10.1097/00003246-199407000-0 0007

43. Veneman T, Mitrakou A, Mokan M, Cryer P, Gerich J. Effect of hyperketonemia and hyperlacticacidemia on symptoms, cognitive dysfunction, and counterregulatory hormone responses during hypoglycemia in normal humans. Diabetes. (1994) 43:1311-7. doi: 10.2337/diab.43.11.1311

44. Thomsen HH, Rittig N, Johannsen M, Møller AB, Jørgensen JO, Jessen $\mathrm{N}$, et al. Effects of 3-hydroxybutyrate and free fatty acids on muscle protein kinetics and signaling during LPS-induced inflammation in humans: anticatabolic impact of ketone bodies. Am J Clin Nutr. (2018) 108:85767. doi: $10.1093 /$ ajen/nqy170

45. Lauritsen KM, Søndergaard E, Svart M, Møller N, Gormsen LC. Ketone body infusion increases circulating erythropoietin and bone marrow glucose uptake. Diabetes Care. (2018) 41:e152-4. doi: 10.2337/dc18-1421

46. Nosadini R, Trevisan R, Fioretto P, Semplicini A, Samà B, Velussi M, et al. Kidney hemodynamics after ketone body and amino acid infusion in normal and IDDM subjects. Diabetes. (1989) 38:75-83. doi: 10.2337/diab.38.1.75

47. Owen OE, Reichard GA Jr. Markus H, Boden G, Mozzoli MA, Shuman $\mathrm{CR}$, et al. Rapid intravenous sodium acetoacetate infusion in man metabolic and kinetic responses J Clin Invest. (1973) 52:2606-16. doi: 10.1172/JCI10 7453

48. Fery F, Balasse EO. Effect of exercise on the disposal of infused ketone bodies in humans. J Clin Endocrinol Metab. (1988) 67:24550. doi: $10.1210 /$ jcem-67-2-245 
49. Kang HC, Chung DE, Kim DW, Kim HD. Early- and late-onset complications of the ketogenic diet for intractable epilepsy. Epilepsia. (2004) 45:111623. doi: 10.1111/j.0013-9580.2004.10004.x

50. Batch JT, Lamsal SP, Adkins M, Sultan S, Ramirez MN. Advantages and disadvantages of the ketogenic diet: a review article. Cureus. (2020) 12:e9639. doi: 10.7759/cureus.9639

51. Bolla AM, Caretto A, Laurenzi A, Scavini M, Piemonti L. Low-carb and ketogenic diets in type 1 and type 2 diabetes. Nutrients. (2019) 11:962. doi: 10.3390/nu11050962

52. Shaikh S, Mohamed MM, Mujeeb A, Shaikh F, Harris B. Euglycemic Diabetic Ketoacidosis Precipitated by a Keto Diet: Importance of Dietary History in Diagnosis. Cureus. (2020) 12:e10199. doi: 10.7759/cureus. 10199

53. Charoensri S, Sothornwit J, Trirattanapikul A, Pongchaiyakul C. Ketogenic diet-induced diabetic ketoacidosis in a young adult with unrecognized type 1 diabetes. Case Rep Endocrinol. (2021) 2021:6620832. doi: 10.1155/2021/6620832

54. Dressler A, Reithofer E, Trimmel-Schwahofer P, Klebermasz K, Prayer D, Kasprian G, et al. Type 1 diabetes and epilepsy: efficacy and safety of the ketogenic diet. Epilepsia. (2010) 51:1086-9. doi: 10.1111/j.1528-1167.2010.02543.x

55. Smith SL, Heal DJ, Martin KF. KTX. 0101: a potential metabolic approach to cytoprotection in major surgery and neurological disorders. CNS Drug Rev. (2005) 11:113-40. doi: 10.1111/j.1527-3458.2005.tb0 0265.x

56. Cahill GF Jr. Veech rl ketoacids? Good medicine? Trans Am Clin Climatol Assoc. (2003) 114:149-61.

57. Neal EG, Chaffe H, Schwartz RH, Lawson MS, Edwards N, Fitzsimmons $\mathrm{G}$, et al. A randomized trial of classical and medium-chain triglyceride ketogenic diets in the treatment of childhood epilepsy. Epilepsia. (2009) 50:1109-17. doi: 10.1111/j.1528-1167.2008.01870.x

58. Clarke K, Tchabanenko K, Pawlosky R, Carter E, Todd King M, MusaVeloso K, et al. Kinetics, safety and tolerability of (R)-3-hydroxybutyl (R)3-hydroxybutyrate in healthy adult subjects. Regul Toxicol Pharmacol. (2012) 63:401-8. doi: 10.1016/j.yrtph.2012.04.008

59. Desrochers S, Dubreuil P, Brunet J, Jetté M, David F, Landau BR, et al. Metabolism of (R,S)-1,3-butanediol acetoacetate esters, potential parenteral and enteral nutrients in conscious pigs. Am J Physiol. (1995) 268:E6607. doi: 10.1152/ajpendo.1995.268.4.E660

Conflict of Interest: The authors declare that the research was conducted in the absence of any commercial or financial relationships that could be construed as a potential conflict of interest.

Publisher's Note: All claims expressed in this article are solely those of the authors and do not necessarily represent those of their affiliated organizations, or those of the publisher, the editors and the reviewers. Any product that may be evaluated in this article, or claim that may be made by its manufacturer, is not guaranteed or endorsed by the publisher.

Copyright (c) 2021 White, Heffernan, Worrall, Grunsfeld and Thomas. This is an open-access article distributed under the terms of the Creative Commons Attribution License (CC BY). The use, distribution or reproduction in other forums is permitted, provided the original author(s) and the copyright owner(s) are credited and that the original publication in this journal is cited, in accordance with accepted academic practice. No use, distribution or reproduction is permitted which does not comply with these terms. 